Service social

\title{
Les personnes âgées, leurs milieux de vie et les pratiques sociales
}

\section{Nicolas Zay}

Volume 34, numéro 1, 1985

Personnes âgées, milieux de vie et pratiques sociales

URI : https://id.erudit.org/iderudit/706247ar

DOI : https://doi.org/10.7202/706247ar

Aller au sommaire du numéro

Éditeur(s)

École de service social de l'Université Laval

ISSN

1708-1734 (numérique)

Découvrir la revue

Citer cet article

Zay, N. (1985). Les personnes âgées, leurs milieux de vie et les pratiques sociales. Service social, 34(1), 11-13. https://doi.org/10.7202/706247ar d'utilisation que vous pouvez consulter en ligne.

https://apropos.erudit.org/fr/usagers/politique-dutilisation/ 


\section{Les personnes âgées, leurs milieux de vie et les pratiques sociales}

Ce numéro de Service social est le troisième, depuis 1973, à être consacré au vieillissement et à la vieillesse. C'est dire que la profession porte un intérêt de plus en plus marqué aux problèmes du troisième âge, comme en témoigne l'esprit de l'avant-propos. Jacques Laforest y lance un défi aux travailleurs sociaux : pour répondre aux vrais besoins des aînés, il leur faut découvrir le voie d'une pratique nouvelle, car ils s'attaquent, dans le champ du service social gérontologique, à des problèmes ponctuels à l'intérieur d'une problématique plus globale. Au lieu de se laisser emprisonner dans l'attitude générale d'une société où il n'y a pas de place pour les personnes âgées, il leur faut aller à contre-courant.

Le premier article, signé par François Béland, est consacré aux représentations que se font respectivement l'État et les usagers des besoins de cette population. Les deux perspectives ne coïncident pas nécessairement. Alors que la demande des usagers reflète un comportement par rapport à une situation vécue, la planification de l'État, souvent teintée de scientisme, est de nature normative et définie à partir d'un point de vue politique. Pour étudier cette différence de représentation, l’auteur a choisi les services de soutien à domicile, où les divergences sont les plus palpables. Sans vouloir subordonner une façon de voir à l'autre, il s'agit pour lui d'en assurer la coexistence.

Lise Darveau-Fournier décrit une expérience dans un milieu de soins prolongés en vue d'y améliorer la qualité de vie des résidents. L'originalité du projet, dont l'objectif était de faire préparer et implanter un programme de formation continue par les aînés eux-mêmes, réside dans l'ampleur de la participation. En plus de diverses catégories d'intervenants, y ont aussi collaboré des usagers et leur famille. Le projet s'insère ainsi dans les tentatives d'associer les usagers à la conception et à la distribution des services qui leur sont destinés.

Lorraine Brissette rapporte les résultats d'une recherche qui a permis d'évaluer l'influence de la fréquentation d'un centre de jour sur le nombre et la nature des relations qu'entretient la personne âgée avec son réseau de support naturel et sur la stratégie qu'elle emploie pour résoudre les problèmes de son quotidien. D'après les 
résultats obtenus dans deux centres de jour, il semblerait que leur fréquentation n'ait pas changé grand-chose à la quantité des relations avec le réseau de support naturel, mais qu'elle ait agi sur la vie intérieure des sujets. Si le centre de jour réussit à répondre à leurs attentes, leur état psychologique peut s'en trouver amélioré et, par voie de conséquence, enrichir leurs rapports avec leur réseau naturel.

Suzanne Moffet, quant à elle, consacre son article à la vie associative des personnes âgées telle qu'elle s'exprime, principalement à travers deux catégories d'associations. Les unes, vouées à l'amélioration de la qualité de vie des ainés, se composent essentiellement de professionnels; les autres, presque exclusivement de personnes âgées qui en assurent la gestion. L'auteur passe en revue la structure de ces groupes aux États-Unis, en France et au Québec, en mettant en évidence les caractéristiques propres à chaque pays. Si la participation associative des aînés s'accroît sensiblement depuis les dix dernières années, il n'en reste pas moins que c'est l'apanage d'une minorité bien portante, plus instruite que la moyenne, financièrement à l'aise et habituée à cette forme d'implication. Quant au nouveau "pouvoir gris", il n'est pas porteur d'une réelle contestation de l'ordre social établi. Ses dirigeants cherchent avant tout à atteindre les autorités pour les induire à assurer un meilleur sort aux aînés et à mieux respecter leurs aspirations. Pour terminer, madame Moffet traite des fonctions de la vie associative, en invitant les intervenants sociaux à travailler en collaboration étroite avec les associations de personnes âgées, non seulement pour enrichir leur propre pratique sociale mais aussi pour établir des canaux de communication plus féconds.

Teresa Sheriff et Rosario Lopez-Tremblay étudient les stratégies auxquelles a recours la personne âgée pour affronter les problèmes quotidiens, et cela dans des cas où la lecture du réel est primordiale pour l'intervention. Elles illustrent le développement de leur thème par le cas de personnes que leur entourage veut placer. Chacune de celles-ci use de son imagination et de son expérience de vie pour résister au placement. En conclusion, les auteurs soulignent la difficulté que présente la collecte des données nécessaires à l'évaluation de la réalité et énumèrent les aspects dont doit tenir compte l'intervenant dans son analyse s'il veut saisir la signification profonde des stratégies.

Verena Bernardin-Haldemann résume une étude sur l'habitat des personnes âgées, à partir d'une constatation à l'effet que plus des trois quarts de celles-ci, au Québec, sont responsables de leur logement. La recherche, de type exploratoire, se concentre sur trois dimensions: l'habitat lui-même, défini à la fois dans ses aspects physique et social; l'espace de vie quotidienne, ou ce qui est traversé par l'individu dans 
ses sorties habituelles; et, enfin, la satisfaction à l'égard de l'habitat. L'auteur met en évidence le rôle de variables comme l'âge, l'accumulation de biens, les ressources économiques, la vie active antérieure et les caractéristiques du quartier. Au plan des politiques et des pratiques sociales, elle pose des interrogations sur l'influence de facteurs comme les ressources collectives, les mécanismes de compensation et l'appropriation de l'espace.

Enfin, Réjean Landry résume les résultats d'une recherche sur les promesses faites par les partis politiques québécois, entre 1960 et 1981, à l'endroit des personnes âgées. Il en conclut que le poids attribué aux promesses dans le programme des partis n'atteint jamais celui que lui attribue le corps électoral. II explique sa constatation par la segmentation progressive de l'électorat et par le fait que, dans l'esprit des dirigeants des partis politiques, l'âge ne peut servir de critère valable à la détermination des priorités.

Pour conclure, soulignons que le numéro que nous présentons met en lumière la variété des préoccupations des intervenants en gérontologie; il illustre aussi la multidisciplinarité de la recherche qui se fait en ce domaine, grâce à laquelle on peut espérer que notre connaissance des nombreux aspects du vieillissement se développe d'une manière harmonieuse et équilibrée.

Nicolas ZAY

Coordonnateur du numéro 